\title{
Dual RNA-Seq Unveils the Role of the Pseudomonas plecoglossicida fliA Gene in Pathogen-Host Interaction with Larimichthys crocea
}

\author{
Yujia Sun ${ }^{1}$, Pin Nie ${ }^{2}{ }^{\oplus}$, Lingmin Zhao ${ }^{1}$, Lixing Huang ${ }^{1}$, Yingxue Qin ${ }^{1}$, Xiaojin $\mathrm{Xu}^{1}$, \\ Jiaonan Zhang ${ }^{3}$ and Qingpi Yan ${ }^{1, *(1)}$ \\ 1 Fisheries College, Key Laboratory of Healthy Mariculture for the East China Sea, Ministry of Agriculture, \\ Jimei University, Xiamen 361021, China; sunyj37@mail2.sysu.edu.cn (Y.S.); 200561000132@jmu.edu.cn (L.Z.); \\ lixinghuang@jmu.edu.cn (L.H.); yxqin@jmu.edu.cn (Y.Q.); xiaojinxu@jmu.edu.cn (X.X.) \\ 2 School of Marine Science and Engineering, Qingdao Agricultural University, Qingdao 266109, China; \\ pinnie@qau.edu.cn \\ 3 Key Laboratory of Special Aquatic Feed for Fujian, Fujian Tianma Technology Company Limited, \\ Fuzhou 350308, China; jolma66@163.com \\ * Correspondence: yanqp@jmu.edu.cn
}

Received: 20 July 2019; Accepted: 8 October 2019; Published: 11 October 2019

\begin{abstract}
In the present study, Larimichthys crocea and Pseudomonas plecoglossicida were selected as a host-pathogen interaction model for teleosts and prokaryotic pathogens. Five shRNAs were designed and synthesized to silence the fliA gene, all of which resulted in pronounced reductions in fliA mRNA; the mutant strain with the best silencing efficiency of $92.16 \%$ was chosen for subsequent analysis. A significant decrease in motility, intracellular survival and escape was observed for the fliA-RNAi strain of P. plecoglossicida, whereby silencing of the fliA gene led to a $30 \%$ decrease in mortality and a four-day delay in the onset of infection in L. crocea. Moreover, silencing of P. plecoglossicida fliA resulted in a significant change in both the pathogen and host transcriptome in the spleens of infected L. crocea. Kyoto Encyclopedia of Genes and Genomes (KEGG) analysis of pathogen transcriptome data showed that silencing fliA resulted in downregulation of 18 flagellum-related genes; KEGG analysis of host transcriptome data revealed that infection with the fliA-RNAi strain caused upregulation of 47 and downregulation of 106 immune-related genes. These pathogen-host interactions might facilitate clearance of P. plecoglossicida by L. crocea, with a significant decrease in fliA-RNAi P. plecoglossicida strain virulence in L. crocea.
\end{abstract}

Keywords: pathogen-host interaction; Larimichthys crocea; Pseudomonas plecoglossicida; fliA; dual RNA-seq

\section{Introduction}

Flagella are an important structure in bacteria and are widely recognized to be involved in their pathogenesis [1,2]. For example, flagella contribute to the virulence of prokaryotic pathogens by promoting adherence [3,4] and the formation of bacterial biofilms [5,6], exporting virulent factors via the type III secretion system $[7,8]$, and activating inflammation via the recognition of Toll-like receptor $5[9,10]$. More than 50 genes are involved in the synthesis of flagella, and they are expressed in a strictly regulated and hierarchical manner [11].

$\sigma^{28}$, which is encoded by the fliA gene, plays a positive regulatory role in flagellar assembly $[12,13]$. $\sigma^{28}$ has been documented to regulate flagellar synthesis in Escherichia coli [14], Campylobacter jejuni [15], Pseudomonas aeruginosa [16], Salmonella typhimurium [17] and Vibrio cholera [18]. Furthermore, knocking out fliA in P. aeruginosa resulted in reduced motility, with decreased colonization in the intestines 
of mice because flagella were not synthesized [19]. In addition, the fliA mutant strain of Legionella pneumophila exhibits reduced motility, weakened biofilm, reduced macrophage infectivity and decreased colonization potential in host cells [20]. Although fliA is well known for its important multiple functions, the role of $f l i A$ in host-pathogen interactions remains unknown, partly due to the limitations of research technology.

The infection process is a fierce battle between the host and pathogen, in which both must strive for success [21]. To win this life-and-death struggle, both pathogen and host must mobilize all available resources, and all changes will be reflected in their respective transcriptome profiles [22]. Dual RNA-seq offers the ability to monitor host-pathogen RNA expression profiles simultaneously [23-27].

Pseudomonas plecoglossicida is known to cause epidemics in cultured fish, such as ayu (Plecoglossus altivelis) [28], large yellow croaker (Larimichthys crocea) [29,30] and rainbow trout (Oncorhynchus mykiss) [31]. The pathogenic mechanism of P. plecoglossicida has attracted considerable attention, and several virulence genes have been recognized and explored [32,33]. In our previous research, the transcriptomes of P. plecoglossicida in the spleens of infected L. crocea were sequenced at 1, 2, 3 and 4 days post-infection (dpi); these data have been deposited in the NCBI database (SRP176599). fliA was noted for its significant upregulation during the entire infection process, and it was hypothesized to play an important role in the host-pathogen interaction.

To explore the role of fliA of P. plecoglossicida in the host-pathogen interaction, the gene was knocked down by RNAi, and phenotypic differences between fliA-RNAi and wild type strains of P. plecoglossicida were analyzed. In addition, dual RNA-seq was performed using the spleens of L. crocea infected by the fliA-RNAi strain or wild type strain of P. plecoglossicida as material, and the data were subjected to a series of bioinformatics analyses. The present study constitutes a new attempt to explore the role of a single gene in the host-pathogen interaction by integrating dual RNA-seq and RNAi.

\section{Results}

\subsection{Construction of the fliA-RNAi Strain of P. plecoglossicida}

Figure 1A shows the RNA-seq and qRT-PCR results of P. plecoglossicida fliA gene expression in the spleen of $L$. crocea during the infection process. Compared with the in vitro culture counterpart, the $f l i A$ gene of was upregulated in the infected spleen at all four sampling times, with the highest expression recorded at $2 \mathrm{dpi}$. The results of qRT-PCR were consistent with those of RNA-seq.
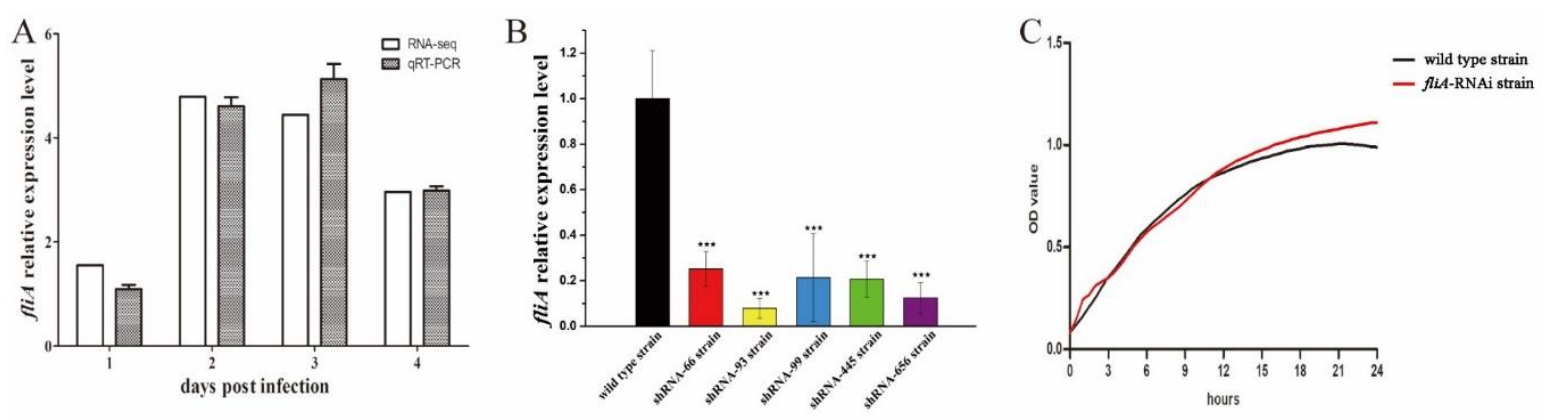

Figure 1. Construction of the fliA-RNAi strain of P. plecoglossicida. (A) Relative expression of fliA at 1, 2, 3 and 4 days post-infection. Each data comes from three samples. (B) Relative expression of fliA in five mutant strains of P. plecoglossicida. Each data comes from three independent experiments. (C) growth curve of P. plecoglossicida. Each data point comes from 12 technical repeats. $\star \star \star p \leq 0.001$. 
According to qRT-PCR results, all five shRNAs designed in this study had a silencing effect on fliA expression in P. plecoglossicida (Figure 1B). The strain containing pCM130/tac-fliA-shRNA-93 exhibited the best $f l i A$-silencing efficiency $(92.16 \%)$ and was chosen as the fliA-RNAi strain for further analysis.

A growth curve was generated to determine whether fliA affects growth. Although fliA was silenced, no significant difference in growth rate between the $f l i A$-RNAi strain and wild type strain of P. plecoglossicida was found when cultured in vitro at $28^{\circ} \mathrm{C}$ (Figure 1C).

\subsection{The Effect of fliA on Motility and Intracellular Survival of P. plecoglossicida}

The soft agar plate test showed that the colony diameters of the wild type strain were considerably larger than those of the fliA-RNAi strain of P. plecoglossicida; in contrast, the colony diameters of the fliA-RNAi strain were smaller than those of the wild type strain (Figure 2A).
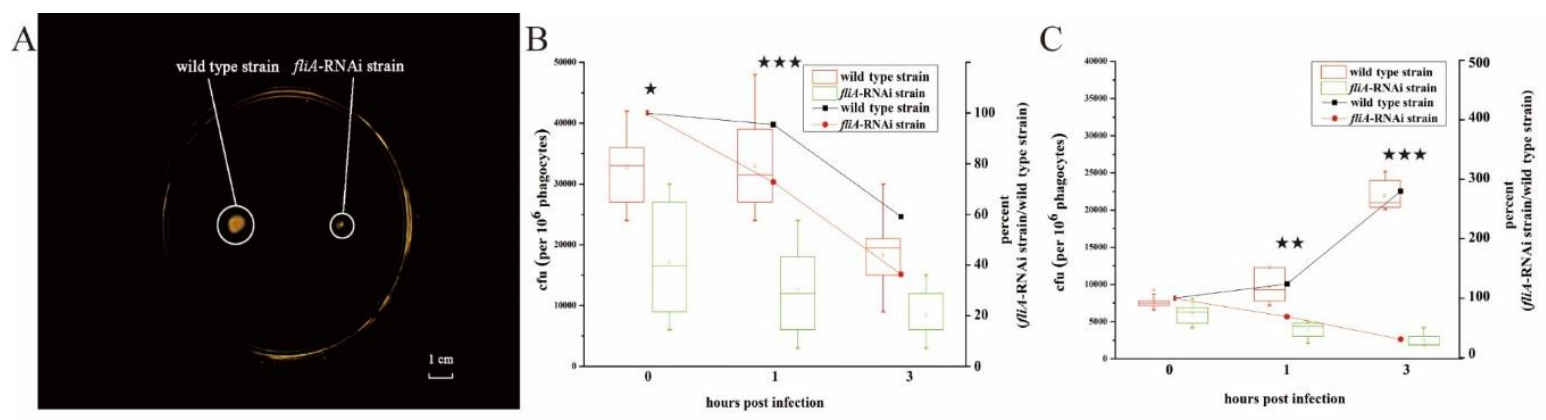

Figure 2. Characterization of the fliA-RNAi strain and wild type strain of P. plecoglossicida. (A) Motility. (B) Survival rate in phagocytes. (C) Escape rate from phagocytes. The right y-axis in Figure 2B,C represent the percent of pathogen cfu number of fliA-RNAi strain group to wild type strain group.

Each data point comes from three samples. $\star p \leq 0.05 ; \star \star p \leq 0.01 ; \star \star \star p \leq 0.001$.

After a 3-h intracellular survival assay, the number of cells of both strains of P. plecoglossicida was significantly reduced. The wild type strain was reduced by $40.91 \%$ and the fliA-RNAi strain by $63.64 \%$, the number of fliA-RNAi cells was significantly reduced compared with wild type (Figure 2B). Conversely, after a 3-h intracellular escape assay, the number of wild type cells increased to $280 \%$, though the number of fliA-RNAi cells was reduced to $30.95 \%$. The escape ability of the fliA-RNAi strain was significantly lower than that of the wild type strain (Figure 2C).

\subsection{The Effect of fliA on P. plecoglossicida Virulence}

Compared with counterparts infected with the wild type strain of P. plecoglossicida, infection of L. crocea with the fliA-RNAi strain resulted in a delay in the time of death by 3 days, and the survival rate increased by $30 \%$ (Figure $3 \mathrm{~A}$ ). 
A
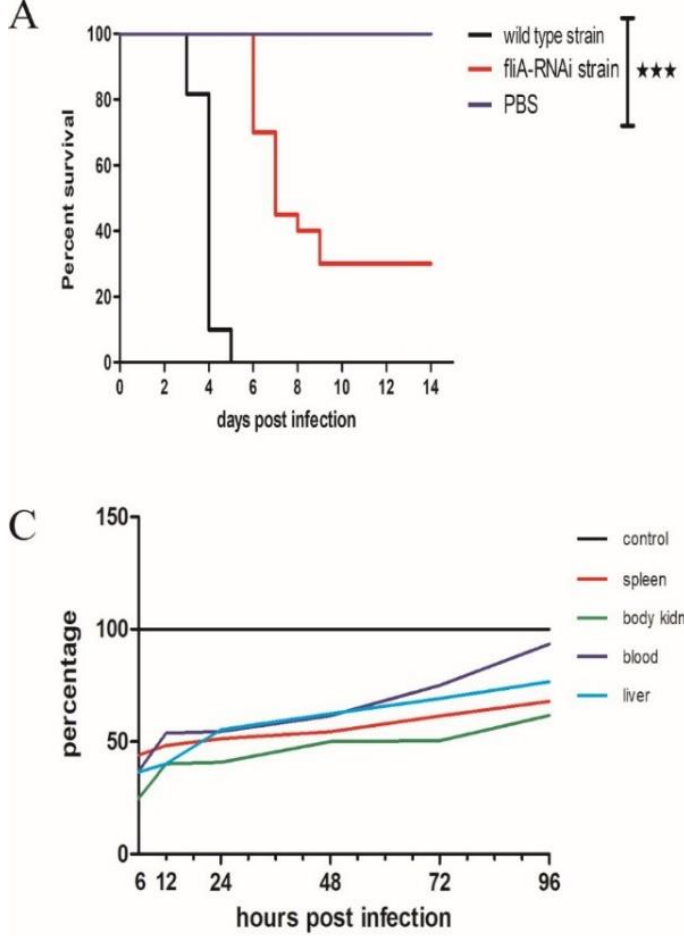

$\mathrm{B}$
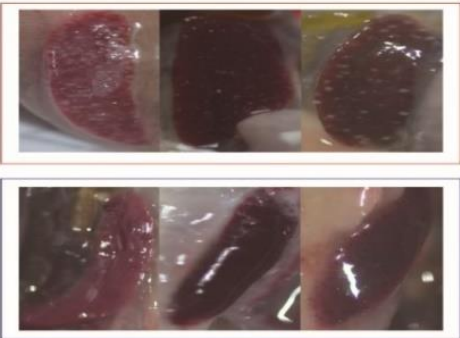

$\begin{aligned} & \operatorname{MiA-RNAi} \\ & \text { strain } 3 \text { dpi }\end{aligned} \mid$

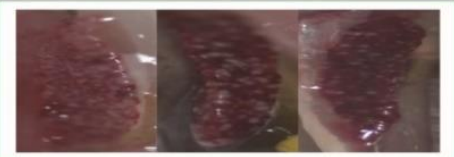

IIA-RNAi strain 7 dpi

$\mathrm{D}$

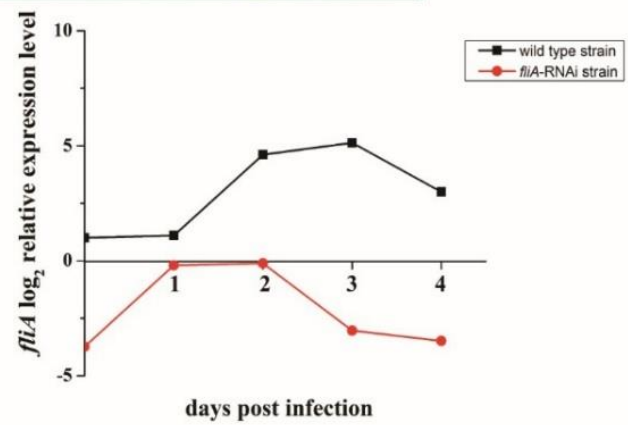

Figure 3. Virulence of the wild type strain and fliA-RNAi strain of P. plecoglossicida. (A) Survival curve of $L$. crocea infected with the wild type strain and fliA-RNAi strain of P. plecoglossicida. (B) Appearance of spleens of infected L. crocea. The upper pictures are spleens infected with the wild type strain of P. plecoglossicida, the middle and lower pictures are spleens infected with the fliA-RNAi strain of P. plecoglossicida. (C) Spatial and temporal distribution of the fliA-RNAi strain of P. plecoglossicida compared to the wild type strain. Each data point comes from three samples. (D) Relative expression of fliA of P. plecoglossicida in L. crocea spleens. Each data point comes from three samples. $\star \star \star p \leq 0.001$.

As piscine spleens infected with P. plecoglossicida are usually covered with abundant white spots [21], dead fish were examined for appearance of spleens. The spleens of $L$. crocea exhibited the typical appearance, with numerous white spots, at 3 days after infection by wild type P. plecoglossicida, though the same appearance did not appear until 7 days after infection by the fliA-RNAi strain (Figure 3B).

We also assessed whether the bearing capacity of the two strains in fish organs was altered, and the difference in distribution of P. plecoglossicida between L. crocea infected with the fliA-RNAi and wild type strains was determined by qRT-PCR. At $6 \mathrm{~h}$ post-infection, the relative quantity of the fliA-RNAi strain in each tissue was lowest, that is, less than half of the quantity of the wild type strain, though the relative quantity of the fliA-RNAi strain increased gradually with the extension of infection time. Moreover, the relative quantity of the fliA-RNAi strain was higher in the blood than in other tissues (Figure 3C).

qRT-PCR was also employed to probe the dynamic expression of $f l i A$ in the two strains of $P$. plecoglossicida during infection. Expression of fliA was upregulated during infection with the wild type strain, increased to a peak at $2 \mathrm{dpi}$, and then decreased gradually. Similarly, the expression of fliA in the $f l i A$-RNAi strain increased at 1 and $2 \mathrm{dpi}$ and then decreased gradually. Overall, expression of fliA in both strains was higher in vivo than in vitro, and expression of $f l i A$ in the fliA-RNAi strain was lower than that in the wild type strain throughout the study (Figure 3D).

Briefly, silencing fliA in P. plecoglossicida resulted in increased survival, delayed time of death and appearance occurrence, reduced quantity distribution in L. crocea and decreased expression of fliA throughout the infection process. 


\subsection{The Effects of fliA on the Transcriptome of Host and Pathogen}

The base distributions of spleen and in vitro-cultured P. plecoglossicida were balanced, and the unknown base $\mathrm{N}$ distribution was lower than that of any other base and in a reasonable range (Figures S1 and S2). The average distribution of base error rates was less than $0.1 \%$, a reasonable range (Figures S3 and S4). To reduce the complexity of data and illustrate the relationship between samples, principal component analysis was carried out, and the result showed that the repeatability of samples was satisfactory (Figures S5 and S6). Twenty-three differentially expressed genes (DEGs) of the host and pathogen transcriptomes were randomly selected and verified by qRT-PCR, and the results were consistent with the transcriptome results (Figure S7).

A total of 22,150 genes were mapped for the host transcriptome. Compared to infection by the wild type strain of P. plecoglossicida, infection by the fliA-RNAi strain resulted in 956 host genes being downregulated and 915 being upregulated in the spleen; the other 20279 genes showed no significant difference (Figure 4A). According to the KEGG database, 47 upregulated DEGs were enriched in three immune-related KEGG pathways, and adjusted $p$-values from high to low indicated involvement of the cytokine-cytokine receptor interaction pathway, lysosome pathway and intestinal immune network and the IgA production pathway (Figure 4B). The lysosome pathway was enriched 19 upregulated genes, encoding glycosidase, protease, phosphatase, sulfatase and lysosomal membrane proteins. Additionally, 106 downregulated DEGs were enriched in 10 KEGG pathways, with 51 DEGs enriched in six immune-related KEGG pathways. Adjusted $p$-values from high to low showed involvement of the cytokine-cytokine receptor interaction pathway, cell adhesion molecule pathway, AGE-RAGE signaling pathway in the diabetic complications pathway, C-type lectin receptor signaling pathway, Toll-like receptor signaling pathway, glycerolipid metabolism pathway, adipocytokine signaling pathway, arachidonic acid metabolism pathway, RIG-I-like receptor signaling pathway and nicotinate and nicotinamide metabolism pathway (Figure 4C).
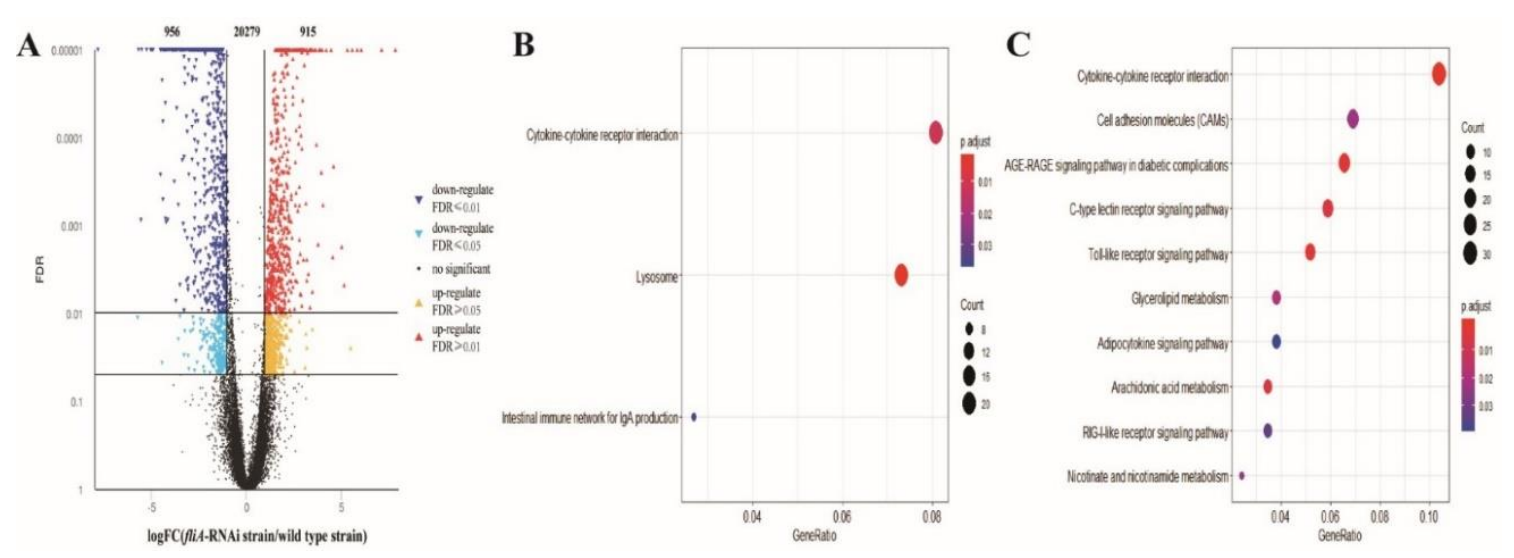

Figure 4. Global characterization of the host transcriptome in the spleen of L. crocea infected by $P$. plecoglossicida. (A) Volcano plot of all genes of the spleen. X-axis: the fold change values of genes of the fliA-RNAi strain infection group/wild type strain infection group. Y-axis: statistical test value (false discovery rate (FDR)), higher values represent more significant differences. Each dot represents a particular gene: red dots denote significantly upregulated genes, blue dots significantly downregulated genes and black dots genes with non-significant differences. (B) KEGG enrichment of upregulated genes. (C) KEGG enrichment of downregulated genes. In B and C, the X-axis shows the GeneRatio, whereby a higher value indicates more genes enriched in the pathway; the Y-axis shows the enriched pathways, and the more red the dot is, the more significant is the pathway. 
For the pathogen transcriptome, a total of 1585 genes were mapped. At $2 \mathrm{dpi}, 1504$ genes were not significantly different, 77 genes were downregulated, and four genes were upregulated in fish infected by the fliA-RNAi strain of P. plecoglossicida compared to the pathogen transcriptome in the spleens of fish infected by the wild type strain (Figure 5A). According to the KEGG database, 30 downregulated DEGs were enriched in three KEGG pathways, including the flagellar assembly pathway, ribosome pathway and RNA degradation pathway, as presented by $p$-values from high to low (Figure 5B).

A

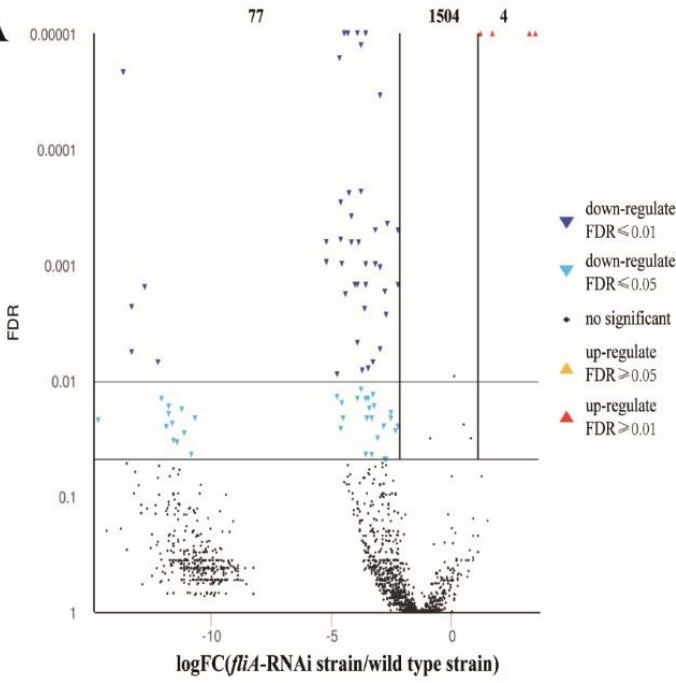

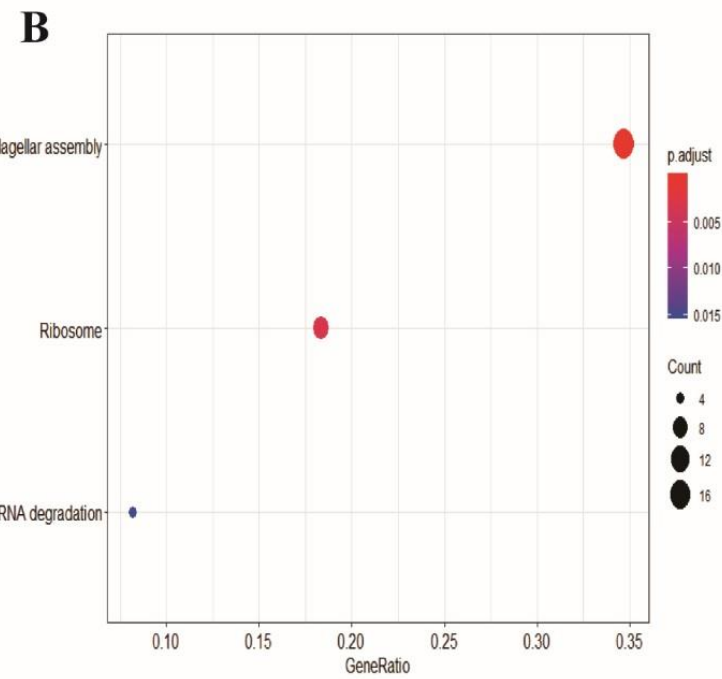

Figure 5. Global characterization of the pathogen transcriptome in the spleen of L. crocea infected by $P$. plecoglossicida. (A) Volcano plot of all genes of P. plecoglossicida. X-axis: the fold change values of genes of the fliA-RNAi strain infection group/wild type strain infection group. Y-axis: the statistical test value (false discovery rate (FDR)), whereby a higher value represents a more significant difference. Each dot represents a particular gene: red dots denote significantly upregulated genes, blue dots significantly downregulated genes and black dots genes with non-significant differences. (B) The X-axis shows the GeneRatio, whereby a higher value indicates more genes enriched in the pathway; the Y-axis shows the enriched pathways, and the more red the dot is, the more significant is the pathway.

\subsection{The Relationship between DEGs of P. plecoglossicida}

RNAi of fliA had a great effect on the expression of genes in the flagellar assembly pathway at 2 dpi. Compared with the group infected by the wild type strain, expression of 18 genes in the flagellar assembly pathway of P. plecoglossicida was downregulated, which included $52.94 \%$ of genes in the flagellar assembly pathway and $73.91 \%$ of flagellar structure genes (Figure 6A). Conversely, no flagellar assembly pathway genes were found to be upregulated. 
A

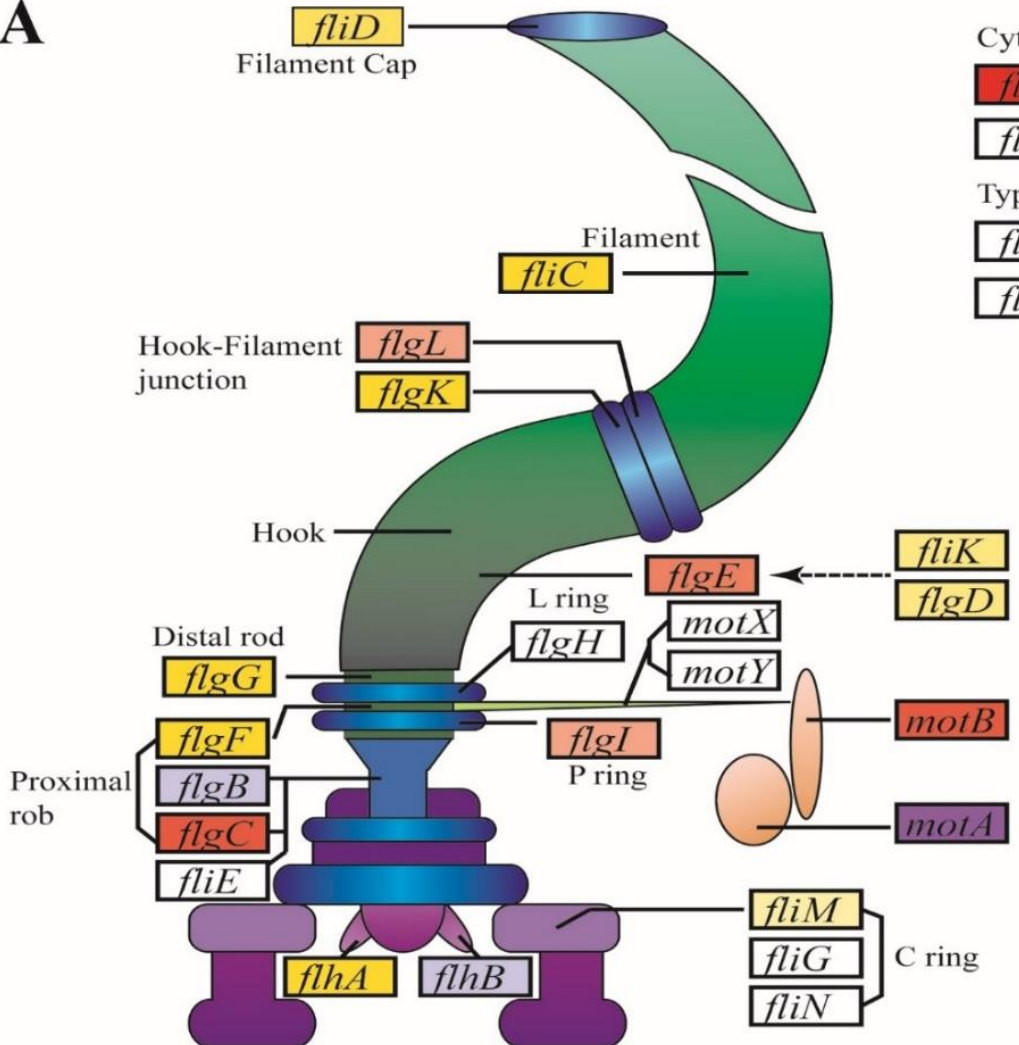

Cytoplasmic Chaperone

flis $f l g N$ fliv

fliT flgA

Type III Secretion System

fliH $f l i I \quad$ fliO

flio $f l g P$ fliR

\section{B}

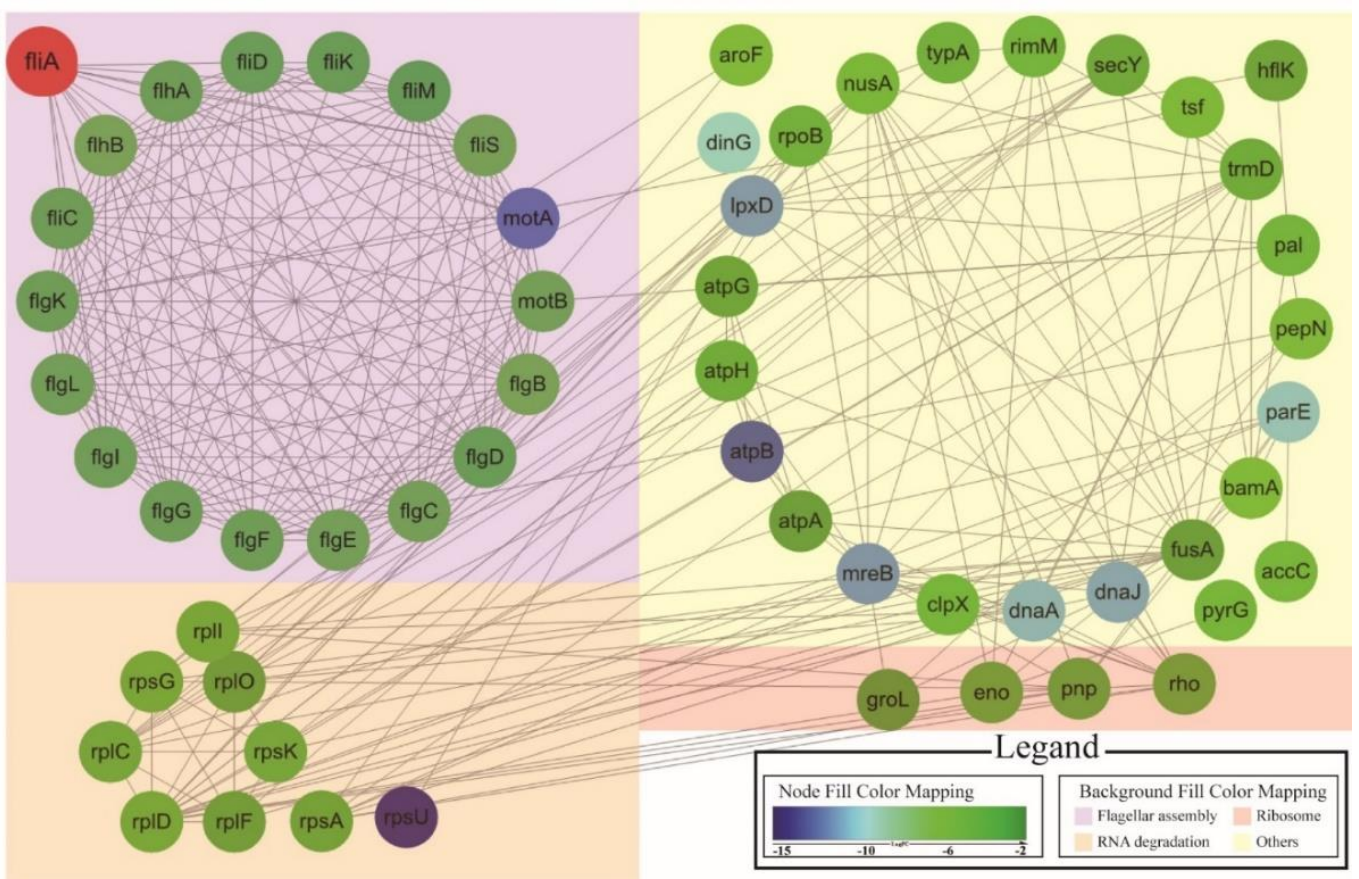

Figure 6. Changes in gene expression in the flagellar assembly pathway and related pathway of P. plecoglossicida. (A) Visualization of the flagellar assembly pathway. Genes with different colors indicate different $\log F C$ values. (B) The relationship between flagellar assembly genes and other genes. Genes range in color from green to blue, whereby a bluer color indicates a greater downregulation of expression. The fliA gene is red. The purple background indicates the flagellar assembly pathway; the orange background indicates the RNA degradation pathway; the red background indicates the ribosome pathway; the yellow background indicates other differentially expressed genes. 
In addition to genes in the flagellar assembly pathway, some genes in other pathways were downregulated by fliA RNA. According to the network constructed by GENEMANIA, fliA controls the flagellar assembly pathway by regulating expression of 13 genes, and fliA regulates non-flagellar genes through genes of the flagellar assembly pathway; flagellar genes indirectly regulate genes of the ribosome pathway, the RNA degradation pathway and other pathways (Figure 6B).

\section{Discussion}

Several genes have been identified to be involved in the pathogenesis of P. plecoglossicida $[29,32-34]$ and other aquatic pathogens $[35,36]$. Accumulating evidence indicates that flagella are an important structure for pathogenic bacteria [37-39]. Prior to this work, fliA had been described as an essential gene for flagellar synthesis and pathogenesis $[19,20]$; therefore, it is necessary to explore the role of $f l i A$ in the host-pathogen interaction of P. plecoglossicida.

In the present study, all five shRNAs caused a significant silencing effect on $f l i A$, though with different efficiencies. The shRNA targeting 93-113 bp downstream of the translation initiation site of the fliA gene had the best silencing efficiency, of $92.16 \%$, and the effect persisted at all sampling times. The result of this silencing established the foundation for further research examining the role of fliA post-infection. Compared to infection of L. crocea by the wild type strain, fliA-RNAi strain infection resulted in a 3-day delay in the time of death, a 30\% reduction in mortality and a 4-day delay of appearances in the spleen. The spleen is a major immune organ that is both specific and non-specific in fish $[40,41]$. In addition, the spleen is the organ displaying typical appearances [42] and maximum amounts of bacteria [34]. Thus, it may be inferred that the spleen is the ideal tissue for researching the interaction of L. crocea and P. plecoglossicida.

Overall, the transcriptome reflects the complete physiological condition of an organism [33]. In a strain of $P$. aeruginosa lacking fliA, eight genes were downregulated and four were upregulated in the flagellar assembly pathway after culture in LB medium [19]. In the present study, silencing fliA in P. plecoglossicida resulted in the identification of 18 genes downregulated in the flagellar assembly pathway $(52.94 \%)$, but no gene was observed to be upregulated. Although many studies have investigated the role of fliA in flagellar synthesis, this report is the first to describe that fliA affects so many flagellar genes during infection. The results indicate that fliA plays a critical role in flagellar assembly, especially in the host. Flagellar and ribosome assembly involve the two largest biomacromolecular complexes in the cell, and they coordinate via the energy cycle [43]. In the present study, the ribosome and RNA degradation pathways of P. plecoglossicida were also downregulated as a result of fliA RNAi, suggesting that fliA plays a more complex role in the regulation of pathogen transcription in the host than previously known.

Dual RNA-seq technology is beginning to be employed to simultaneously monitor global changes between host and pathogen [23,26,27]. In the present study, during infection, the deficient strain of fliA showed blocked flagellar synthesis, and expression of Toll-like receptor 5 (tlr5) was decreased, as expected. tlr5 specifically recognizes flagellin $[10,44]$ and can induce expression of several proinflammatory cytokine genes, such as $i l-1 \beta$ and $\operatorname{tnf} \alpha[45,46]$, the latter of which is activated by arachidonic acid in Kupper cells [47]. In the present study, fliA RNAi in P. plecoglossicida resulted in downregulation of $i l-1 \beta$ and $t n f \alpha$ expression in the host, as well as $i l-6, i l-8$ and $i l-12$, which are known to be regulated by Toll-like receptor 4 (tlr4) [48-51]. In addition, the arachidonic acid metabolism pathway was downregulated, indicating a negative regulatory mechanism between $\operatorname{tnf} \alpha$ and arachidonic acid. Moreover, a coexpression relationship has been documented for arachidonic acid metabolism and nicotinamide metabolism, which may explain the downregulation of nicotinate and nicotinamide metabolism pathways [52]. The nicotinamide and glycerolipid metabolism pathways are both damaged in chronic progressive heart failure, though the mechanism has not been full elucidated [53]. Flagella facilitate the survival of bacteria within macrophages or their escape from macrophages $[2,54]$. In the present study, RNAi of fliA resulted in the reduced ability of $P$. plecoglossicida to survive and escape from phagocytes as well as a decrease in motility. Moreover, fliA RNAi led to upregulation of some 
host genes related to lysosomes, including glycosidase, protease, phosphatase and sulfatase, which facilitate host clearance of pathogens.

\section{Materials and Methods}

\subsection{Bacterial Strains and Culture Conditions}

P. plecoglossicida NZBD9 was isolated from the spleen of a L. crocea individual with "Visceral White Spot Disease" [42]. The E. coli DH5 $\alpha$ strain was purchased from TransGen Biotech (Beijing, China). The bacteria were routinely cultured in Luria Bertani (LB) medium at $18^{\circ} \mathrm{C}$ (P. plecoglossicida) and $37^{\circ} \mathrm{C}$ (E. coli). LB medium containing $1 \mu \mathrm{g} / \mathrm{mL}$ tetracycline was used to select the strain containing the plasmid pCM130/tac.

\subsection{Construction of the RNAi Strain}

pCM130/tac [34] was used to construct the P. plecoglossicida RNAi strain according to a previously described method $[55,56]$. Five siRNA sequences targeting the fliA gene were designed by BLOCK-iTTM RNAi Designer (http://rnaidesigner.thermofisher.com/rnaiexpress/setOption.do?designOption=shrna\& pid $=708587103220684543$ ) and synthetized by Shanghai Generay Biotech Co., Ltd. (Shanghai, China; Table S1). First, the pCM130/tac vector was linearized using the restriction enzymes Nsil-HF and BsrGI-HF (New England Biolabs, Ipswich, MA, USA) and ligated to annealed shRNA with T4 DNA ligase. Second, the recombinant plasmid was transformed into E. coli DH5a by heat shock, extracted using EasyPure Plasmid MiniPrep Kit (TransGen Biotech, Beijing, China) and electroporated into P. plecoglossicida. Each strain was cultured to an $\mathrm{OD}_{600} 0.5 \pm 0.1$, and the level of fliA mRNA was detected by quantitative real-time (qRT)-PCR for three independent experiments.

\subsection{Quantitative Real-Time PCR ( $q R T-P C R)$}

qRT-PCR was performed using QuantStudio 6 Flex (Life Technologies) as previously described [57]. All primer sequences used in the present study are provided in Table S2. Each qRT-PCR experiment was repeated five times.

\subsection{Growth Curve Determination}

The wild type strain or fliA-RNAi strain of P. plecoglossicida was cultured in LB medium at $28{ }^{\circ} \mathrm{C}$ until the OD reached $0.4 \sim 0.6$ and then diluted to $0.2 \pm 0.01$. Next, $50 \mu \mathrm{L}$ aliquots of diluted bacterial suspension was added to twelve wells of microtiter plate which preload $150 \mu \mathrm{L}$ sterile LB medium and incubated at $28^{\circ} \mathrm{C}$. The values of $\mathrm{OD}_{600}$ of each well were read using a SYNERGY H1 microplate reader (BioTec, Dorset, UK).

\subsection{Swimming Motility Assay}

The swimming motility assay was performed using plates half-filled LB medium with $0.3 \%$ agar. The wild type and fliA-RNAi strains of P. plecoglossicida were incubated until the value of $\mathrm{OD}_{600}$ reached $0.4 \sim 0.6$; the culture medium was then diluted to an $\mathrm{OD}$ of $0.3 \pm 0.01$, and $1 \mu \mathrm{L}$ was inoculated onto the centre of each plate $[37,58]$. The assay was technical repeated three times.

\subsection{Intracellular Survival and Escape Assay}

Healthy L. crocea individuals were purchased from Ningde (Fujian, China) and anaesthetized with 4-ethyl-amino-benzocaine before head kidney sampling. The tissues were pushed through a 100-mesh nylon screen and suspended in L-15 medium (Biological Industries, Kibbutz Beit- Haemek, Israel) containing $100 \mathrm{IU}$ streptomycin/penicillin (S/P)/mL and $2 \%$ fetal calf serum (FCS). The tissues were transferred to a 200-mesh nylon screen and centrifuged at $400 \times g$ for $20 \mathrm{~min}$, and $2 \mathrm{~mL}$ L-15 medium was added. The cell suspension was layered onto a 34\%/51\% discontinuous Percoll (Amersham Pharmacia Biotech, New York, NY, USA) density gradient with a syringe and centrifuged at $400 \times g$ for $20 \mathrm{~min}$ 
at $4{ }^{\circ} \mathrm{C}$. Next, the cells in the layer above the 34\%/51\% interface were collected, washed twice with phosphate-buffered saline (PBS) and resuspended in L-15 medium with 10\% FCS, 100 IU S/P/mL. The cells were then incubated at $28^{\circ} \mathrm{C}$ for $3 \mathrm{~h}$, after which non-adherent cells were removed by washing with L-15, and the monolayers were collected. The cell suspension was added to $2 \times 10^{6}$ cells $/ \mathrm{mL}$ in L-15 medium with 10\% FCS and $100 \mathrm{IU} \mathrm{S} / \mathrm{P} / \mathrm{mL}$ and transferred to six-well plates at $1.5 \mathrm{~mL} /$ well. The pathogen was added in a volume of $1.5 \mathrm{~mL}$ to each plate in a ratio of 1 cell to 100 pathogen cells, which were washed twice with PBS. The cells were infected with the wild type or fliA-RNAi strain incubated with $250 \mu \mathrm{g} / \mathrm{mL}$ tetracycline or ofloxacin at $28^{\circ} \mathrm{C}$ for $20 \mathrm{~min}$, and infected cells were washed with PBS, centrifuged at 400 $\times g$ and $20 \mathrm{~min}$, and resuspended in $3 \mathrm{~mL} \mathrm{L-15}$ medium containing 10\% FCS and 100 IU S/P/mL. The 3-mL cell solution was divided into three parts on average for three checkpoints. Each part was centrifuged at $400 \times g$ for $20 \mathrm{~min}$, and the supernatant was diluted 10 times and inoculated onto plates. The infected cells at the bottom of the tube were lysed with $\mathrm{ddH}_{2} \mathrm{O}$, diluted 100 times and inoculated onto plates to determine the survival rate [59]. The assay was repeated three times.

\subsection{L. crocea Infection Experiments}

All fish experiments were executed strictly following the recommendations of 'National Institutes of Health Guide for the Care and Use of Laboratory Animals'. The animal protocols were conducted strictly according to the Animal Ethics Committee of Jimei University (Acceptance NO JMULAC201159, date of approval: 20 December 2011).

Weight-matched healthy L. crocea were maintained at $18{ }^{\circ} \mathrm{C}$ to adapt to the experimental temperature for one week. The wild type and fliA-RNAi strains of P. plecoglossicida were incubated at $18{ }^{\circ} \mathrm{C}$ until reaching an $\mathrm{OD}_{600}$ value of $0.4 \sim 0.6$ and were then centrifuged and diluted with PBS.

One infection experiment was carried out for survival rate assay. 120 L. crocea were randomly divided into six groups, three groups for wild type strain of P. plecoglossicida challenge; the other three groups for RNAi strain challenge. Each fish was jnjected intrapleurally with $10^{4}$ colony-forming units of the wild type or fliA-RNAi strain of P. plecoglossicida per gram fish (cfu/g); 60 fish injected with PBS were used as a negative control. L. crocea mortality was recorded every day.

Another infection experiment was carried out for sampling. 60 fish were challenged with wild type or fliA-RNAi strain of P. plecoglossicida as described above. For spatial and temporal distribution assays, three random fish from both wild type and fliA-RNAi strain infection groups were used to sample the spleen, liver, trunk kidney and blood at 6, 12, 24, 48, 72 and 96 hpi. For the dual RNA-seq assay, nine fish from both wild type and fliA-RNAi strain infection groups were used to sample the spleen at $2 \mathrm{dpi}$. Three spleens were pooled as one independent sample.

\subsection{DNA and RNA Extraction}

Genomic DNA was extracted from the spleens, livers and trunk kidneys of infected L. crocea using EasyPure Marine Animal Genomic DNA Kit (TransGen Biotech, Beijing, China). Genomic DNA was extracted from the blood using EasyPure Blood Genomic DNA Kit (TransGen Biotech, Beijing, China).

Total RNA was isolated from the spleens, livers, blood and trunk kidneys using the TRIzol reagent (Invitrogen, Carlsbad, CA, USA), and the coexisting DNA was digested by Turbo DNA-free DNase (Ambion, Austin, TX, USA). The quality of the RNA obtained was assessed using an Agilent 2100 Bioanalyzer (Agilent Technologies, Santa Clara, CA, USA), and rRNA was removed by Ribo-Zero rRNA Removal Kit (Epicentre, Madison, WI, USA). cDNA synthesis was conducted with TransScript All-in-One First-Strand cDNA Synthesis SuperMix for PCR (TransGen Biotech, Beijing, China). 


\subsection{Illumina Sequencing}

The cDNA libraries of spleens were amplified by Phusion DNA polymerase (New England Biolabs, Ipswich, MA, USA), and an Agilent 2100 Bioanalyzer (Agilent Technologies, City of Santa Clara, CA, USA) was employed to validate the library quality. Finally, sequencing was performed by Majorbio Biotech Co., Ltd. (Shanghai, China) using the Illumina HiSeq 4000 sequencing platform.

\subsection{Processing and Mapping of Reads}

Filtering and quality control of the raw Illumina reads were performed by SeqPrep (https://github. com/jstjohn/SeqPrep), Sickle (https://github.com/najoshi/sickle) and Fastx-Toolkit (http://hannonlab. cshl.edu/fastx_toolkit/) with default settings. The clean data were mapped to the NB2011 strain genome of P. plecoglossicida (NCBI RefSeq accession numbers: NZ_ASJX00000000.1) by Bowtie2 [60]. Mapped reads were mapped to the P. plecoglossicida genome and the L. crocea genome.

\subsection{Analysis of Differential Gene Expression}

Identification of differentially expressed genes was performed using the $\mathrm{R}$ package edgeR (version

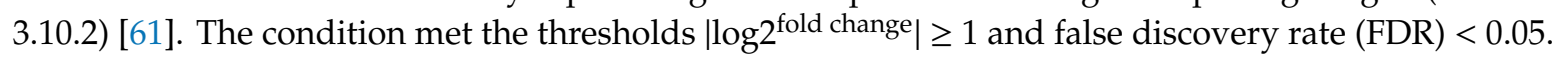

\subsection{KEGG Enrichment Analysis}

KEGG enrichment analysis was performed using the $\mathrm{R}$ package 'clusterProfiler' [62]. The thresholds pvalueCutoff $\leq 0.05$ and qvalueCutoff $\leq 0.05$ were applied to identify differentially expressed KEGG pathways.

\subsection{Statistical Analyses}

All data are presented as the mean \pm standard deviation (SD) based on at least three independent experiments. Dunnett's test was used to calculate different experimental data using SPSS 17.0 software (Chicago, IL, USA). GraphPad Prism software was employed for survival rate analysis.

\subsection{Data Access}

The RNA sequencing reads data were deposited in the GenBank SRA database under the accession number SRP183207.

\section{Conclusions}

In summary, silencing of the fliA gene inhibited the synthesis and assembly of P. plecoglossicida's flagella, enabling P. plecoglossicida to be engulfed by L. crocea phagocytes and leading to upregulation of phagocytosis-related genes; however, the relationship between the decrease in the number of extracellular pathogens and the downregulation of several immune pathway genes needs to be explained by further experiment (Figure 7). These host-pathogen interactions facilitate removal of $P$. plecoglossicida by L. crocea, and a significant decrease in the virulence to L. crocea of the fliA-RNAi strain of P. plecoglossicida was observed. 


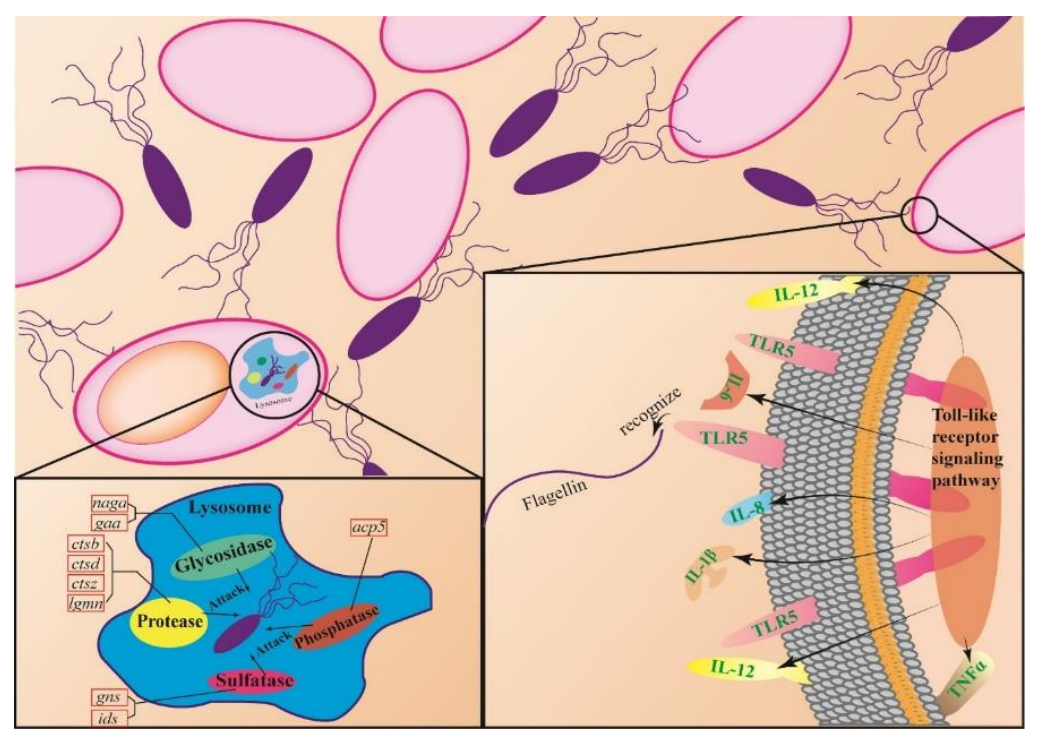

Figure 7. Role of P. plecoglossicida fliA during interaction with L. crocea. The schematic diagram of host pathogen interaction was drawn based on the experimental results of this paper and the literatures.

Supplementary Materials: Supplementary materials can be found at http://www.mdpi.com/2076-2607/7/10/443/s1. Figure S1. Eukaryotic base percentage composition reads. Figure S2. Prokaryotic base percentage composition reads. Figure S3. Eukaryotic distribution of mean error. Figure S4. Prokaryotic distribution of mean error. Figure S5. Eukaryotic PCA analysis. Figure S6. Prokaryotic PCA analysis. Figure S7. Verification dual transcriptome data by qRT-PCR. Table S1. shRNA sequences used in this study. Table S2. Primer sequences used in this study.

Author Contributions: Eight authors contributed to the article. Q.Y. and P.N. conceived of the experiments. Y.S., and L.Z. conducted the experiments. All authors assisted in the collection and interpretation of data. Y.S., P.N. and Q.Y. wrote the manuscript.

Funding: This work was supported by the National Natural Science Foundation of China under contract No. 31672694 and the open fund of Fujian Province Key Laboratory of Special Aquatic Formula Feed under contract No. 2019-01.

Conflicts of Interest: The authors declare no conflict of interest.

\section{References}

1. Macnab, R.M. How bacteria assemble flagella. Annu. Rev. Microbiol. 2003, 57, 77-100. [CrossRef] [PubMed]

2. Qin, Y.; Lin, G.; Chen, W.; Huang, B.; Huang, W.; Yan, Q. Flagellar motility contributes to the invasion and survival of Aeromonas hydrophila in Anguilla japonica macrophages. Fish Shellfish Immunol. 2014, 39, $273-279$. [CrossRef] [PubMed]

3. Girón, J.A.; Torres, A.G.; Freer, E.; Kaper, J.B. The flagella of enteropathogenic Escherichia coli mediate adherence to epithelial cells. Mol. Microbiol. 2002, 44, 361-379. [CrossRef] [PubMed]

4. Tasteyre, A.; Barc, M.C.; Collignon, A.; Boureau, H.; Karjalainen, T. Role of FliC and FliD flagellar proteins of Clostridium difficile in adherence and gut colonization. Infect. Immun. 2001, 69, 7937-7940. [CrossRef] [PubMed]

5. Lemon, K.P.; Higgins, D.E.; Kolter, R. Flagellar motility is critical for Listeria monocytogenes biofilm formation. J. Bacteriol. 2007, 189, 4418-4424. [CrossRef] [PubMed]

6. O'toole, G.A.; Kolter, R. Flagellar and twitching motility are necessary for Pseudomonas aeruginosa biofilm development. Mol. Microbiol. 1998, 30, 295-304. [CrossRef]

7. Young, G.M.; Schmiel, D.H.; Miller, V.L. A new pathway for the secretion of virulence factors by bacteria: The flagellar export apparatus functions as a protein-secretion system. Proc. Natl. Acad. Sci. USA 1999, 96, 6456-6461. [CrossRef]

8. Komoriya, K.; Shibano, N.; Higano, T.; Azuma, N.; Yamaguchi, S.; Aizawa, S.-I. Flagellar proteins and type III-exported virulence factors are the predominant proteins secreted into the culture media of Salmonella typhimurium. Mol. Microbiol. 1999, 34, 767-779. [CrossRef] 
9. Feuillet, V.; Medjane, S.; Mondor, I.; DeMaria, O.; Pagni, P.P.; Galán, J.E.; Flavell, R.A.; Alexopoulou, L. Involvement of Toll-like receptor 5 in the recognition of flagellated bacteria. Proc. Natl. Acad. Sci. USA 2006, 103, 12487-12492. [CrossRef]

10. Qin, G.; Zhang, Y.; Zhang, B.; Wang, X.; Yin, J.; Lin, Q. Seahorse TLR5 gene responses to Vibrio vulnificus infection, which in combination with scuticociliates causes heavy reductions in seahorse aquaculture. J. Fish Dis. 2018, 41, 1933-1936. [CrossRef]

11. Duan, Q.; Zhou, M. Flagella and bacterial pathogenicity. J. Basic Microbiol. 2013, 53, 1-8. [CrossRef] [PubMed]

12. Lonetto, M.; Gribskov, M. The sigma 70 family: Sequence conservation and evolutionary relationships. J. Bacteriol. 1992, 174, 3843. [CrossRef] [PubMed]

13. Chilcott, G.S.; Hughes, K.T. Coupling of flagellar gene expression to flagellar assembly in Salmonella enterica Serovar Typhimurium and Escherichia coli. Microbiol. Mol. Biol. Rev. 2000, 64, 694-708. [CrossRef] [PubMed]

14. Arnosti, D.N.; Chamberlin, M.J. Secondary sigma factor controls transcription of flagellar and chemotaxis genes in Escherichia coli. Proc. Natl. Acad. Sci. USA 1989, 86, 830-834. [CrossRef]

15. Konkel, M.E.; Klena, J.D.; Rivera-Amill, V.; Monteville, M.R.; Biswas, D.; Raphael, B.; Mickelson, J. Secretion of virulence proteins from Campylobacter jejuni is dependent on a functional flagellar export apparatus. J. Bacteriol. 2004, 186, 3296-3303. [CrossRef]

16. Dasgupta, N.; Wolfgang, M.C.; Goodman, A.L.; Arora, S.K.; Jyot, J.; Lory, S.; Ramphal, R. A four-tiered transcriptional regulatory circuit controls flagellar biogenesis in Pseudomonas aeruginosa. Mol. Microbiol. 2003, 50, 809-824. [CrossRef]

17. Ohnishi, K.; Kutsukake, K.; Suzuki, H.; Iino, T. Gene fliA encodes an alternative sigma factor specific for flagellar operons in Salmonella typhimurium. Mol. Gen. Genet. MGG 1990, 221, 139-147. [CrossRef]

18. Prouty, M.G.; Correa, N.E.; Klose, K.E. The novel $\sigma 54$-and $\sigma 28$-dependent flagellar gene transcription hierarchy of Vibrio cholerae. Mol. Microbiol. 2001, 39, 1595-1609. [CrossRef]

19. Lo, Y.L.; Chen, C.L.; Shen, L.; Chen, Y.-C.; Wang, Y.-H.; Lee, C.-C.; Wang, L.-C.; Chuang, C.-H.; Janapatla, R.P.; Chiu, C.-H.; et al. Characterization of the role of global regulator FliA in the pathophysiology of Pseudomonas aeruginosa infection. Res. Microbiol. 2018, 169, 135-144. [CrossRef]

20. Appelt, S.; Heuner, K. The flagellar regulon of Legionella-A Review. Front. Cell. Infect. Microbiol. 2017, 7, 454. [CrossRef]

21. Sun, Y.; Luo, G.; Zhao, L.; Huang, L.; Qin, Y.; Su, Y.; Yan, Q. Integration of RNAi and RNA-seq reveals the immune responses of Epinephelus coioides to sigX gene of Pseudomonas plecoglossicida. Front. Immunol. 2018, 9 , 1624. [CrossRef] [PubMed]

22. Zhang, B.; Luo, G.; Zhao, L.; Huang, L.; Qin, Y.; Su, Y.; Yan, Q. Integration of RNAi and RNA-seq uncovers the immune responses of Epinephelus coioides to L321_RS19110 gene of Pseudomonas plecoglossicida. Fish Shellfish Immunol. 2018, 81, 121-129. [CrossRef] [PubMed]

23. Westermann, A.J.; Förstner, K.U.; Amman, F.; Barquist, L.; Chao, Y.; Schulte, L.N.; Müller, L.; Reinhardt, R.; Stadler, P.F.; Vogel, J. Dual RNA-seq unveils noncoding RNA functions in host-pathogen interactions. Nature 2016, 529, 496. [CrossRef] [PubMed]

24. Aprianto, R.; Slager, J.; Holsappel, S.; Veening, J.-W. Time-resolved dual RNA-seq reveals extensive rewiring of lung epithelial and pneumococcal transcriptomes during early infection. Genome Biol. 2016, 17, 198. [CrossRef]

25. Nuss, A.M.; Beckstette, M.; Pimenova, M.; Schmühl, C.; Opitz, W.; Pisano, F.; Heroven, A.K.; Dersch, P. Tissue dual RNA-seq allows fast discovery of infection-specific functions and riboregulators shaping host-pathogen transcriptomes. Proc. Natl. Acad. Sci. USA 2017, 114, E791-E800. [CrossRef]

26. Sun, Y.; Zhuang, Z.; Wang, X.; Huang, H.; Fu, Q.; Yan, Q. Dual RNA-seq reveals the effect of the flgM gene of Pseudomonas plecoglossicida on the immune response of Epinephelus coioides. Fish Shellfish Immunol. 2019, 87, 515-523. [CrossRef]

27. Zhang, B.; Zhuang, Z.; Wang, X.; Huang, H.; Fu, Q.; Yan, Q. Dual RNA-Seq reveals the role of a transcriptional regulator gene in pathogen-host interactions between Pseudomonas plecoglossicida and Epinephelus coioides. Fish Shellfish Immunol. 2019, 87, 778-787. [CrossRef]

28. Nishimori, E.; Kitatsukamoto, K.; Wakabayashi, H. Pseudomonas plecoglossicida sp. nov. the causative agent of bacterial haemorrhagic ascites of ayu, Plecoglossus altivelis. Int. J. Syst. Evol. Microbiol. 2000, 50, 83-89. [CrossRef] 
29. Huang, L.; Zuo, Y.; Jiang, Q.; Su, Y.; Qin, Y.; Xu, X.; Zhao, L.; Yan, Q. A metabolomic investigation into the temperature-dependent virulence of Pseudomonas plecoglossicida from large yellow croaker (Pseudosciaena crocea). J. Fish Dis. 2019, 42, 431-446. [CrossRef]

30. Zhang, J.; Wang, Y.; Guo, H.; Mao, Z.; Ge, C. Identification and characterization of a phospholipase A1 activity type three secreted protein, PP _ExoU from Pseudomonas plecoglossicida NB 2011, the causative agent of visceral granulomas disease in large yellow croaker (Larimichthys crocea). J. Fish Dis. 2017, 40, 831-840. [CrossRef]

31. Akaylı, T.; Çanak, Ö.; Bașaran, B. A new Pseudomonas species observed in cultured young rainbow trout (Oncorhynchus mykiss Walbaum, 1792): Pseudomonas plecoglossicida. BİBAD Biyol. Bilimleri Arastırma Dergisi 2011, 4, 107-111.

32. Huang, L.; Liu, W.; Jiang, Q.; Zuo, Y.; Su, Y.; Zhao, L.; Qin, Y.; Yan, Q. Integration of transcriptomic and proteomic approaches reveals the temperature-dependent virulence of Pseudomonas plecoglossicida. Front. Cell. Infect. Microbiol. 2018, 8, 207. [CrossRef] [PubMed]

33. Tang, R.; Luo, G.; Zhao, L.; Huang, L.; Qin, Y.; Xu, X.; Su, Y.; Yan, Q. The effect of a LysR-type transcriptional regulator gene of Pseudomonas plecoglossicida on the immune responses of Epinephelus coioides. Fish Shellfish Immunol. 2019, 89, 420-427. [CrossRef] [PubMed]

34. Luo, G.; Xu, X.; Zhao, L.; Qin, Y.; Huang, L.; Su, Y.; Yan, Q. clpV is a key virulence gene during in vivo Pseudomonas plecoglossicida infection. J. Fish Dis. 2019, 42, 991. [CrossRef]

35. Wang, Y.; Wang, X.; Ali, F.; Li, Z.; Fu, Y.; Yang, X.; Lin, W.; Lin, X. Comparative extracellular proteomics of Aeromonas hydrophila reveals iron-regulated secreted proteins as potential vaccine candidates. Front. Immunol. 2019, 10, 256. [CrossRef]

36. Yao, Z.J.; Guo, Z.; Wang, Y.; Li, W.; Fu, Y.; Lin, Y.; Lin, W.; Lin, X. Integrated succinylome and metabolome profiling reveals crucial role of S-ribosylhomocysteine lyase in Qquorum Ssensing and metabolism of Aeromonas hydrophila. Mol. Cell. Proteom. 2019, 18, 200-215. [CrossRef]

37. Qin, Y.; Lin, G.; Chen, W.; Xu, X.; Yan, Q. Flagellar motility is necessary for Aeromonas hydrophila adhesion. Microb. Pathog. 2016, 98, 160-166. [CrossRef]

38. Herlihey, F.A.; Clarke, A.J. Controlling autolysis during flagella insertion in Gram-negative bacteria. In Protein Reviews; Springer: Singapore, 2016; Volume 925, pp. 41-56. [CrossRef]

39. Guerry, P. Campylobacter flagella: Not just for motility. Trends Microbiol. 2007, 15, 456-461. [CrossRef]

40. Dalmo, R.A.; Ingebrigtsen, K.; Bøgwald, J. Non-specific defence mechanisms in fish, with particular reference to the reticuloendothelial system (RES). J. Fish Dis. 1997, 20, 241-273. [CrossRef]

41. Fänge, R.; Nilsson, S. The fish spleen: Structure and function. Experientia 1985, 41, 152-158. [CrossRef]

42. Hu, J.; Zhang, F.; Xu, X.; Su, Y.; Qin, Y.; Ma, Y.; Zhang, Y.; Han, K.; Yan, Q. Isolation, identification and virulence of the pathogen of white-spots diseases in internal organs of Pseudosciaena crocea. Oceanol. Limnol. Sin. 2014, 2, 409-417. [CrossRef]

43. Lemke, J.J.; Durfee, T.; Gourse, R.L. DksA and ppGpp directly regulate transcription of the Escherichia coli flagellar cascade. Mol. Microbiol. 2009, 74, 1368-1379. [CrossRef] [PubMed]

44. Gao, Q.; Yue, Y.; Min, M.; Peng, S.; Shi, Z.; Sheng, W.; Zhang, T. Characterization of TLR5 and TLR9 from silver pomfret (Pampus argenteus) and expression profiling in response to bacterial components. Fish Shellfish Immunol. 2018, 80, 241-249. [CrossRef] [PubMed]

45. Medzhitov, R. Toll-like receptors and innate immunity. Nat. Rev. Immunol. 2001, 1, 135. [CrossRef]

46. Vijay-Kumar, M.; Aitken, J.D.; Carvalho, F.A.; Cullender, T.C.; Mwangi, S.; Srinivasan, S.; Sitaraman, S.V.; Knight, R.; Ley, R.E.; Gewirtz, A.T. Metabolic syndrome and altered gut microbiota in mice lacking Toll-like receptor 5. Science 2010, 328, 228-231. [CrossRef]

47. Cubero, F.J.; Nieto, N. Arachidonic acid stimulates TNF $\alpha$ production in Kupffer cells via a reactive oxygen species-pERK1/2-Egr1-dependent mechanism. Am. J. Physiol.-Gastrointest. Liver Physiol. 2012, 303, G228-G239. [CrossRef]

48. Ozinsky, A.; Underhill, D.M.; Fontenot, J.D.; Hajjar, A.M.; Smith, K.D.; Wilson, C.B.; Schroeder, L.; Aderem, A. The repertoire for pattern recognition of pathogens by the innate immune system is defined by cooperation between toll-like receptors. Proc. Natl. Acad. Sci. USA 2000, 97, 13766-13771. [CrossRef]

49. Chow, J.C.; Young, D.W.; Golenbock, D.T.; Christ, W.J.; Gusovsky, F. Toll-like receptor-4 mediates lipopolysaccharide-induced signal transduction. J. Biol. Chem. 1999, 274, 10689-10692. [CrossRef] 
50. Machida, K.; Cheng, K.T.; Sung, V.M.H.; Levine, A.M.; Foung, S.; Lai, M.M. Hepatitis C virus induces toll-like receptor 4 expression, leading to enhanced production of beta interferon and interleukin-6. J. Virol. 2006, 80, 866-874. [CrossRef]

51. Radstake, T.R.; Roelofs, M.F.; Jenniskens, Y.M.; Oppers-Walgreen, B.; Van Riel, P.L.C.M.; Barrera, P.; Joosten, L.A.B.; Berg, W.B.V.D. Expression of Toll-like receptors 2 and 4 in rheumatoid synovial tissue and regulation by proinflammatory cytokines interleukin-12 and interleukin-18 via interferon- $\gamma$. Arthritis Rheum. 2004, 50, 3856-3865. [CrossRef]

52. Ma, Y.; Bao, Y.; Wang, S.; Li, T.; Chang, X.; Yang, G.; Meng, X. Anti-inflammation effects and potential mechanism of saikosaponins by regulating nicotinate and nicotinamide metabolism and arachidonic acid metabolism. Inflammation 2016, 39, 1453-1461. [CrossRef] [PubMed]

53. Wang, Y.; Li, C.; Chuo, W.; Liu, Z.; Ouyang, Y.; Li, D.; Han, J.; Wu, Y.; Guo, S.; Wang, W. Integrated proteomic and metabolomic analysis reveals the NADH-mediated TCA cycle and energy metabolism disorders based on a new model of chronic progressive heart failure. Mol. BioSyst. 2013, 9, 3135-3145. [CrossRef] [PubMed]

54. Sano, G.I.; Takada, Y.; Goto, S.; Maruyama, K.; Shindo, Y.; Oka, K.; Matsui, H.; Matsuo, K. Flagella facilitate escape of Salmonella from oncotic macrophages. J. Bacteriol. 2007, 189, 8224-8232. [CrossRef] [PubMed]

55. Choi, K.H.; Schweizer, H.P. mini-Tn7 insertion in bacteria with single attTn7 sites: Example Pseudomonas aeruginosa. Nat. Protoc. 2006, 1, 153-161. [CrossRef]

56. Darsigny, M.; Babeu, J.P.; Seidman, E.G.; Gendron, F.-P.; Levy, E.; Carrier, J.; Perreault, N.; Boudreau, F. Hepatocyte nuclear factor- $4 \alpha$ promotes gut neoplasia in mice and protects against the production of reactive oxygen species. Cancer Res. 2010, 70, 9423-9433. [CrossRef]

57. Guo, L.; Huang, L.; Su, Y.; Qin, Y.; Zhao, L.; Yan, Q. $\sec A$, secD, secF, yajC, and yidC contribute to the adhesion regulation of Vibrio alginolyticus. MicrobiolOpen 2018, 7, e00551. [CrossRef]

58. Torabi Delshad, S.; Soltanian, S.; Sharifiyazdi, H.; Bossier, P. Effect of quorum quenching bacteria on growth, virulence factors and biofilm formation of Yersinia ruckeri in vitro and an in vivo evaluation of their probiotic effect in Rainbow trout. J. Fish Dis. 2018, 41, 1429-1438. [CrossRef]

59. Yamamoto, F.Y.; Sutili, F.J.; Hume, M.; Gatlin, D.M., III. The effect of $\beta$-1, 3-glucan derived from Euglena gracilis (Algamune ${ }^{\mathrm{TM}}$ ) on the innate immunological responses of Nile tilapia (Oreochromis niloticus L.). J. Fish Dis. 2018, 41, 1579-1588. [CrossRef]

60. Langmead, B.; Salzberg, S.L. Fast gapped-read alignment with Bowtie 2. Nat. Methods 2012, 9, 357. [CrossRef]

61. Robinson, M.D.; McCarthy, D.J.; Smyth, G.K. edgeR: A Bioconductor package for differential expression analysis of digital gene expression data. Bioinformatics 2010, 26, 139-140. [CrossRef]

62. Yu, G.; Wang, L.-G.; Han, Y.; He, Q.-Y. ClusterProfiler: An R package for comparing biological themes among gene clusters. Omics A J. Integr. Biol. 2012, 16, 284-287. [CrossRef] [PubMed] 